\title{
Spectral Characterization of Anomalous Diffusion of a Periodic Piecewise Linear Intermittent Map
}

\author{
S. Tasaki \\ Advanced Institute for Complex Systems and Department of Applied Physics, \\ School of Science and Engineering, Waseda University, \\ 3-4-1 Okubo, Shinjuku-ku, Tokyo 169-8555, Japan \\ P. Gaspard \\ Center of Nonlinear Phenomena and Complex Systems, \\ Université Libre de Bruxelles, Campus Plaine C. P. 231, \\ B-1050 Brussels, Belgium
}

\begin{abstract}
For a piecewise linear version of the periodic map with anomalous diffusion, the evolution of statistical averages of a class of observables with respect to piecewise constant initial densities is investigated and generalized eigenfunctions of the Frobenius-Perron operator are explicitly derived. The evolution of the averages is controlled by real eigenvalues as well as continuous spectra terminating at the unit circle. Appropriate scaling limits are shown to give a normal diffusion if the reduced map is in the stationary regime with normal fluctuations, a Lévy flight if the reduced map is in the stationary regime with Lévy-type fluctuations and a transport of ballistic type if the reduced map is in the non-stationary regime.
\end{abstract}

Key words: generalized spectral decomposition, generalized eigenfunctions, intermittent map, anomalous diffusion, Lévy flight

PACS: 


\section{INTRODUCTION}

The Frobenius-Perron (FP) operator is a powerful tool to investigate statistical properties of dynamical systems[1,2,3]. For strongly chaotic maps where all trajectories are exponentially unstable such as expanding and hyperbolic systems, correlation decays exponentially and the decay rates, known as PollicottRuelle resonances [4,5], are given as the logarithms of the zeros of the dynamical zeta function. Note that the zeta function is essentially the Fredholm determinant of the FP operator and its zeros are eigenvalues of the FP operator in a generalized sense. Similar characterization was observed for periodic strongly chaotic maps with deterministic diffusion (see $[2,3,6,7]$ and references therein): There, because of the periodicity of the system, the FP operator is decomposed into Fourier components and each component admits wavenumber-dependent eigenvalues including hydrodynamic modes of diffusion. Moreover, the corresponding eigenfunctions have fractality, which is closely related to the underlying phase space structure and the transport coefficients[8].

The method of the dynamical zeta function is extended to certain non-hyperbolic maps with marginal fixed points $[9,10]$. For those maps, the power-law decay of correlation and/or the anormalous growth of the mean square displacement are obtained from the zeta function. The main difference in the zeta function between the non-hyperbolic and hyperbolic maps is the existence of a cut terminating at the unit circle in the former case. In other words, the FP operator of the non-hyperbolic map has a continuous spectrum terminating at the unit circle. However, its spectral structures such as the eigenfunctions associated with the continuous spectrum are not fully understood. Recently, as a first step towards the full spectral characterization of intermittent chaos in non-hyperbolic maps, we have obtained generalized eigenfunctions of the FP operator[11] for a piecewise linear version of the Pomeau-Manneville map[12] introduced by Artuso[10]. We continue the study of Ref.[11] for non-hyperbolic maps with anomalous diffusion and, in this paper, we investigate generalized eigenvalues and the associated eigenfunctions of the FP operator for a piecewise linear version of the map studied by Geisel, Nierwetberg and Zacherl[13]. The mean square displacement of this map spreads faster than the normal diffusion, or it exhibits super-diffusion. Maps with sub-diffusion such as that studied by Geisel and Thomae[14] will be discussed elsewhere.

Note that anomalous transports such as the Lévy diffusion and Lévy flight are known to appear in a variety of natural systems and have been extensively studied (see [15] and refereces therein). and still new maps with anomalous diffusion are proposed[16,17]. Then, it is worthwhile to develop a dynamical systems approach because it would provide a microscopic understanding of the anomalous transports. This is one of the objectives of this work. 
The paper is organized as follows. In Sec.2, we describe a model and classes of observables and initial densities. Evolution of statistical averages of observables is investigated in Sec.3 and generalized eigenfunctions of the FP operator are derived. The behavior of the wavenumber-dependent eigenvalues responsible for the anomalous diffusion is investigated in Sec.4. As one of the applications, 'macroscopic' transports are considered in appropriate scaling limits. The normal diffusion is derived if the reduced map is in the stationary regime with normal fluctuations and Lévy flights are obtained if the reduced map is in the stationary regime with Lévy-type fluctuations. And if the reduced map is in the non-stationar regime, a new transport equation is shown to be obtained. Conclusions are drawn in Sec. 5 .

\section{Piecewise Linear Geisel-Nierwetberg-Zacherl Map}

One of the simplest maps with super-diffusion is that introduced by Geisel, Nierwetberg and Zacherl[13]. Here we consider its piecewise linear version $\tilde{\phi}$. The map is defined on the whole real line (Fig.1 (a)): $\tilde{\phi}: \mathbf{R} \rightarrow \mathbf{R}$ as

$$
\tilde{\phi}(x)= \begin{cases}\phi(x-[x])+[x]-1, & \text { if } x-[x] \leq 1 / 2 \\ \phi(x-[x])+[x]+1 . & \text { if } x-[x]>1 / 2\end{cases}
$$

The symbol $[x]$ stands for the smallest integer which does not exceed $x$, and $\phi:[0,1] \rightarrow[0,1]$ is the reduced map (cf. Fig.1 (b)) defined as follows:

$$
\phi(y)= \begin{cases}\eta_{k}\left(y-\xi_{k}\right)+\xi_{k-1}, & \left(\xi_{k} \leq y \leq \xi_{k-1}\right) \\ \eta_{k}\left(y-1+\xi_{k}\right)+1-\xi_{k-1}, & \left(1-\xi_{k-1} \leq y \leq 1-\xi_{k}\right)\end{cases}
$$

where $k=1,2, \cdots$ and the variables $\xi_{k}$ and $\eta_{k}$ are given by

$$
\begin{gathered}
\xi_{k-1}-\xi_{k}=\frac{1}{2 \zeta(\beta)}\left(\frac{1}{k}\right)^{\beta}(k=1,2, \cdots), \quad \xi_{0}=\frac{1}{2} \\
\eta_{k}= \begin{cases}\frac{\xi_{k-2}-\xi_{k-1}}{\xi_{k-1}-\xi_{k}}=\left(\frac{k}{k-1}\right)^{\beta}, & (k=2,3, \cdots) \\
\frac{1}{1-2 \xi_{1}}=\zeta(\beta), & (k=1)\end{cases}
\end{gathered}
$$

In the above, $\beta>1$ is a parameter and $\zeta(s)=\sum_{n=1}^{\infty} n^{-s}$ is the Riemann zeta function. The map $\phi$ has marginal fixed points $y=0$ and $y=1$ where the map is approximated, respectively, as $\phi(y) \simeq y+c_{0} y^{\beta /(\beta-1)}$ and $\phi(y) \simeq$ $y-c_{0}(1-y)^{\beta /(\beta-1)}$ with a positive constant $c_{0}$. When $\beta>2$, the map $\phi$ is in the stationary regime and, when $\beta \leq 2$, it is in the non-stationary regime. 


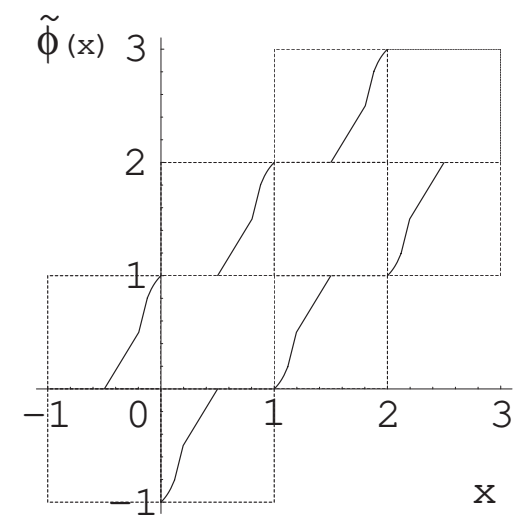

(a)

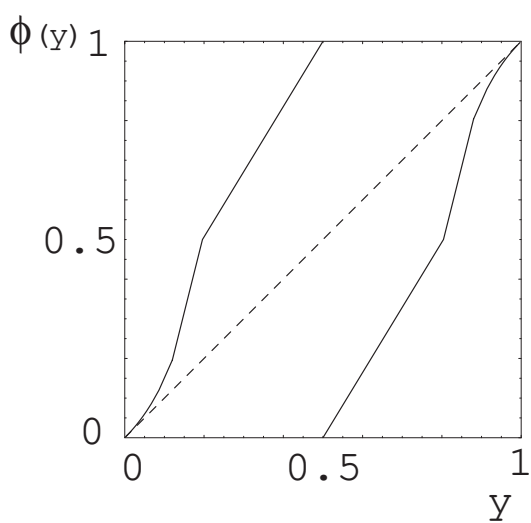

(b)

Fig. 1. (a) The piecewise linear Geisel-Nierwetberg-Zacherl map $\widetilde{\phi}$ and (b) its reduced map $\phi(\beta=2)$.

Moreover, fluctuations in the stationary regime are normal when $\beta>3$ and of Lévy-type when $3 \geq \beta>2[18]$. Those fluctuations lead to anomalous diffusion. Note that a similar piecewise linear map was studied by Artuso[10].

The FP operator $\hat{P}$ and its adjoint $\hat{P}^{*}$ are defined as

$$
\begin{aligned}
\hat{P} \rho(x) & =\int_{-\infty}^{+\infty} d y \delta(x-\tilde{\phi}(y)) \rho(y) \\
\hat{P}^{*} A(x) & =A(\tilde{\phi}(x)) .
\end{aligned}
$$

Thanks to the periodicity of the system, it is convenient to introduce the Fourier representation with respect to the integer part of the coordinate $[x]$ :

$$
\hat{A}(q, y)=\sum_{n=-\infty}^{+\infty} e^{i n q} A(n+y), \quad \hat{\rho}(q, y)=\sum_{n=-\infty}^{+\infty} e^{i n q} \rho(n+y)
$$

where $y \in[0,1)$. Then, the average value of an observable $A$ at time $t$ with respect to an initial density $\rho$ is given by

$$
\left(A, \hat{P}^{t} \rho\right) \equiv\left(\hat{P}^{* t} A, \rho\right)=\int_{-\pi}^{+\pi} \frac{d q}{2 \pi} \int_{0}^{1} d y \hat{A}_{t}(q, y)^{*} \hat{\rho}(q, y)
$$

where $(A, \rho)$ is the inner product:

$$
(A, \rho)=\int_{-\infty}^{+\infty} d x A(x) \rho(x)=\int_{-\pi}^{\pi} \frac{d q}{2 \pi} \int_{0}^{1} d y \hat{A}(q, y)^{*} \hat{\rho}(q, y)
$$


and $\hat{A}_{t}(q, y)^{*}$ is the Fourier transform of $\hat{P}^{t *} A$ obeying the recursion relation:

$$
\hat{A}_{t+1}(q, y)^{*}= \begin{cases}e^{-i q} \hat{A}_{t}(q, \phi(y))^{*}, & 0 \leq y<\frac{1}{2} \\ e^{i q} \hat{A}_{t}(q, \phi(y))^{*}, & \frac{1}{2} \leq y<1\end{cases}
$$

In order to extract information about generalized eigenfunctions of the FP operator, we consider the time evolution of the averages of observables with respect to a class of initial densities.

The Fourier transform of observables $\hat{A}(q, y)$ are assumed to be smooth at the origin and at $y=1$ in the sense that they behave as $\hat{A}(q, y)=\hat{A}(q, 0)+$ $a_{1}(q) y+\mathrm{O}\left(y^{\beta /(\beta-1)}\right)$ near $y=0$ and as $\hat{A}(q, y)=\hat{A}(q, 1)+a_{2}(q)(y-1)+$ $\mathrm{O}\left((1-y)^{\beta /(\beta-1)}\right)$ near $y=1$, where $a_{1}$ and $a_{2}$ correspond, respectively, to $\left.\partial_{y} \hat{A}(q, y)\right|_{y=0}$, and $\left.\partial_{y} \hat{A}(q, y)\right|_{y=1}$. More precisely, $\hat{A}(q, y)$ is a bounded function such that there exist functions $a_{1}(q)$ and $a_{2}(q)$ of $q$ and inequalities

$$
\begin{aligned}
& \left|\hat{A}(q, y)-\hat{A}(q, 0)-a_{1}(q) y\right| \leq K|y|^{\beta /(\beta-1)} \\
& \left|\hat{A}(q, y)-\hat{A}(q, 1)-a_{2}(q)(y-1)\right| \leq K^{\prime}|1-y|^{\beta /(\beta-1)}
\end{aligned}
$$

hold for $0 \leq y \leq 1$ and $-\pi \leq q \leq \pi$, where $K$ and $K^{\prime}$ are positive constants. Let $X_{O}$ be a set of such functions, then it is a Banach space with respect to the norm:

$$
\begin{aligned}
\|A\|_{O}= & \sum_{j=1,2}\left\|a_{j}\right\|_{\infty}+\|\hat{A}\|_{\infty}+\sup _{q, 0<y \leq 1} \frac{\left|\hat{A}(q, y)-\hat{A}(q, 0)-a_{1}(q) y\right|}{|y|^{\beta /(\beta-1)}} \\
& +\sup _{q, 0 \leq y<1} \frac{\left|\hat{A}(q, y)-\hat{A}(q, 1)-a_{2}(q)(y-1)\right|}{|1-y|^{\beta /(\beta-1)}}
\end{aligned}
$$

where $\|\cdot\|_{\infty} \equiv \sup _{q, x}|\cdot|$. The space $X_{O}$ is invariant with respect to the adjoint of the FP operator $\hat{P}^{*}$ : namely, if $A \in X_{O}$, then $\hat{P}^{*} A \in X_{O}$. Note that since $X_{O}$ contains a space $C^{2}[-\pi, \pi] \times[0,1]$ of twice continuously differentiable functions and $C^{2}[-\pi, \pi] \times[0,1]$ is dense in the Hilbert space $L^{2}[-\pi, \pi] \times[0,1]$ of square integrable functions, $X_{O}$ is dense in $L^{2}[-\pi, \pi] \times[0,1]$.

It was shown $[4,5]$ that the spectrum of the FP operator of expanding maps is controlled by the smoothness of densities. In this proof, the positivity of the Lyapunov exponents plays an essential role. However, the map $\phi$ does not enjoy such a property and the general situation is not known. So, we restrict ourselves to a narrow class of piecewise constant initial densities in order to derive explicit expressions of the generalized eigenfunctions of the FP 
operator. More precisely, we consider a set $X_{D}$ of initial densities $\rho$ given by

$$
\hat{\rho}(q, y)= \begin{cases}\widetilde{\rho}_{k}^{-}(q), & \left(\xi_{k} \leq y \leq \xi_{k-1}\right) \\ \widetilde{\rho}_{k}^{+}(q), & \left(1-\xi_{k-1} \leq y \leq 1-\xi_{k}\right)\end{cases}
$$

where $k=1,2, \cdots$ and

$$
\tilde{\rho}_{k}^{ \pm}(q)=\sum_{l=0}^{+\infty} \rho_{l}^{ \pm}(q)\left(\frac{k}{k+l}\right)^{\beta} \quad \text { with } \quad \sum_{l=0}^{+\infty}\left\|\rho_{l}^{ \pm}\right\|_{\infty} \theta^{l}<+\infty
$$

for some constant $\theta>1$. The space $X_{D}$ is a Banach space with respect to the norm

$$
\|\rho\|_{D}=\sum_{l=0}^{+\infty}\left\{\left\|\rho_{l}^{+}\right\|_{\infty}+\left\|\rho_{l}^{-}\right\|_{\infty}\right\} \theta^{l}
$$

and is invariant under the action of the FP operator $\hat{P}$ : namely, if $\rho \in X_{D}$, then $\hat{P} \rho \in X_{D}$. Note that, in contrast to the space $X_{O}$ of observables, the space $X_{D}$ of densities is not dense in the Hilbert space $L^{2}[-\pi, \pi] \times[0,1]$ of square integrable functions.

\section{Evolution of statistical averages of observables}

\subsection{Matrix elements of the resolvent}

In this section, we investigate the evolution of the average $\left(A, \hat{P}^{t} \rho\right)$ of an observable $A$ with respect to a piecewise constant initial density $\rho$. We begin with the Neumann series of the matrix element of the resolvent:

$$
\left(A, \frac{1}{z \mathbf{1}-\hat{P}} \rho\right)=\sum_{t=0}^{+\infty} \frac{1}{z^{t+1}}\left(A, \hat{P}^{t} \rho\right)
$$

where $\mathbf{1}$ is the identity operator. In terms of

$$
B_{k}^{-}(t, q)=\int_{\xi_{k}}^{\xi_{k-1}} d y \hat{A}_{t}(q, y)^{*}, \quad \text { and } \quad B_{k}^{+}(t, q)=\int_{1-\xi_{k-1}}^{1-\xi_{k}} d y \hat{A}_{t}(q, y)^{*}
$$

each term of the series can be rewritten as 


$$
\left(A, \hat{P}^{t} \rho\right)=\int_{-\pi}^{\pi} \frac{d q}{2 \pi} \sum_{k=0}^{\infty}\left\{\widetilde{\rho}_{k}^{+}(q) B_{k}^{+}(t, q)+\widetilde{\rho}_{k}^{-}(q) B_{k}^{-}(t, q)\right\},
$$

and, hence,

$$
\left(A, \frac{1}{z \mathbf{1}-\hat{P}} \rho\right)=\int_{-\pi}^{+\pi} \frac{d q}{2 \pi} \sum_{k=1}^{+\infty}\left\{\widetilde{\rho}_{k}^{+}(q) \hat{B}_{k}^{+}(q, z)+\widetilde{\rho}_{k}^{-}(q) \hat{B}_{k}^{-}(q, z)\right\}
$$

where $\hat{B}_{k}^{ \pm}(z, q) \equiv \sum_{t=0}^{\infty} B_{k}^{ \pm}(t, q) / z^{t+1}$. The right-hand side of (20) is calculated as follows: For example, when $k \geq 2$, eq.(2) gives recursion relations of $B_{k}^{ \pm}(t, q)$ :

$$
\begin{aligned}
B_{k}^{-}(t+1, q) & =\int_{\xi_{k}}^{\xi_{k-1}} d y e^{-i q} \hat{A}_{t}\left(q, \eta_{k}\left(y-\xi_{k}\right)+\xi_{k-1}\right)^{*} \\
& =\frac{e^{-i q}}{\eta_{k}} \int_{\xi_{k-1}}^{\xi_{k-2}} d y \hat{A}_{t}(q, y)^{*}=\frac{e^{-i q}}{\eta_{k}} B_{k-1}^{-}(t, q), \\
B_{k}^{+}(t+1, q) & =\frac{e^{i q}}{\eta_{k}} B_{k-1}^{+}(t, q),
\end{aligned}
$$

and, hence, we obtain

$$
\hat{B}_{k}^{ \pm}(q, z)=\frac{1}{z} B_{k}^{ \pm}(q, 0)+\frac{e^{ \pm i q}}{z \eta_{k}} \hat{B}_{k-1}^{ \pm}(q, z)
$$

Similarly, one has

$$
\hat{B}_{1}^{ \pm}(q, z)=\frac{1}{z} B_{1}^{ \pm}(q, 0)+\frac{e^{ \pm i q}}{z \eta_{1}} \sum_{k=1}^{+\infty} \hat{B}_{k}^{\mp}(q, z)
$$

The recursion relations (23) and (24) give

$$
\begin{aligned}
& \sum_{k=1}^{+\infty} \tilde{\rho}_{k}^{ \pm}(q) \hat{B}_{k}^{ \pm}(q, z)=\Xi^{ \pm}(q, z)+\frac{B_{1}^{ \pm}(q, 0) \Psi^{ \pm}(q, z)}{z} \\
& +\frac{\Psi^{ \pm}(q, z)}{\Omega(q, z)}\left\{\frac{Z\left(z e^{ \pm i q}\right)}{(z \zeta(\beta))^{2}} \Phi^{ \pm}(q, z)+\frac{e^{ \pm i q}}{z \zeta(\beta)} \Phi^{\mp}(q, z)\right\}
\end{aligned}
$$

where $\Xi^{ \pm}(q, z), \Phi^{ \pm}(q, z), \Psi^{ \pm}(q, z), \Omega(q, z)$ and $Z(z)$ are defined by: 


$$
\begin{aligned}
\Xi^{ \pm}(q, z) & =\sum_{l=2}^{\infty} \frac{l^{\beta} B_{l}^{ \pm}(q, 0)}{z}\left[\left(z e^{\mp i q}\right)^{l-1} \Psi^{ \pm}(q, z)-\sum_{k=1}^{l-1} \frac{\widetilde{\rho}_{k}^{ \pm}(q)}{k^{\beta}}\left(z e^{\mp i q}\right)^{l-k}\right] \\
\Phi^{ \pm}(q, z) & =\sum_{l=1}^{\infty} \sum_{k=0}^{\infty}\left(\frac{e^{ \pm i q}}{z}\right)^{k}\left(\frac{l}{k+l}\right)^{\beta} \frac{B_{l}^{ \pm}(q, 0)}{z} \\
\Psi^{ \pm}(q, z) & =\sum_{k=1}^{\infty} \frac{\widetilde{\rho}_{k}^{ \pm}(q)}{k^{\beta}}\left(\frac{e^{ \pm i q}}{z}\right)^{k-1} \\
\Omega(q, z) & =1-\frac{Z\left(z e^{i q}\right) Z\left(z e^{-i q}\right)}{(z \zeta(\beta))^{2}} \\
Z(z) & =\sum_{k=1}^{\infty} \frac{z^{-k+1}}{k^{\beta}}
\end{aligned}
$$

Infinite series in (26), (27), (28) and (30) are absolutely convergent for $|z|>1$ and, thus, are analytic there. In addition, because of (17) and the analyticity of the resolvent in $|z|>1$, one finds

$$
\left(A, \hat{P}^{t} \rho\right)=\oint_{|z|=r} \frac{d z}{2 \pi i} z^{t}\left(A, \frac{1}{z \mathbf{1}-\hat{P}} \rho\right)=\int_{-\pi}^{+\pi} \frac{d q}{2 \pi} \oint_{|z|=r} \frac{d z}{2 \pi i} z^{t} R(q, z)
$$

where $R(q, z) \equiv \sum_{k=1}^{\infty}\left\{\widetilde{\rho}_{k}^{+}(q) \hat{B}_{k}^{+}(q, z)+\widetilde{\rho}_{k}^{-}(q) \hat{B}_{k}^{-}(q, z)\right\}$ and the integration path is a counter-clockwise circle centered at $z=0$ with radius $r(>1)$.

\subsection{Analytical properties of individual functions}

Here we derive analytical continuations of $\Phi^{ \pm}(q, z), \Psi^{ \pm}(q, z)$ and $Z(z)$ into the unit disk $|z|<1$. With the aid of the formula $\Gamma(\beta) / k^{\beta}=\int_{0}^{\infty} d s s^{\beta-1} e^{-k s}$, the series expression of $\Phi^{ \pm}$can be rewritten as

$$
\begin{aligned}
\Phi^{ \pm}(q, z) & =\sum_{l=1}^{\infty} \frac{l^{\beta} B_{l}^{ \pm}(q, 0)}{z \Gamma(\beta)} \int_{0}^{\infty} d s s^{\beta-1} e^{-l s} \sum_{k=0}^{\infty}\left(\frac{e^{-s}}{z e^{\mp i q}}\right)^{k} \\
& =\sum_{l=1}^{\infty} \frac{l^{\beta} B_{l}^{ \pm}(q, 0)}{e^{ \pm i q} \Gamma(\beta)} \int_{0}^{\infty} d s \frac{s^{\beta-1} e^{-l s}}{z e^{\mp i q}-e^{-s}}
\end{aligned}
$$

The above calculations are justified because the summation converges uniformly in $s$ provided $|z|>1$.

We observe that, as a result of an inequality for bounded observables $A$ : $l^{\beta}\left|B_{l}^{ \pm}(q, 0)\right| \leq l^{\beta}\left(\xi_{l-1}-\xi_{l}\right)\|A\|_{\infty}=\|A\|_{\infty} /(2 \zeta(\beta))$, the estimate 


$$
\sum_{l=1}^{\infty}\left|\frac{l^{\beta} B_{l}^{ \pm}(q, 0)}{e^{ \pm i q} \Gamma(\beta)} \int_{0}^{\infty} d s \frac{s^{\beta-1} e^{-l s}}{z e^{\mp i q}-e^{-s}}\right| \leq \frac{\|A\|_{\infty}}{d\left(z e^{\mp i q},[0,1]\right)}
$$

holds where $d(\xi,[0,1])$ is the distance between $\xi$ and the real interval $[0,1]$. It is obvious that each term of $\Phi^{ \pm}$in the right-hand side of (32) is analytic except a cut on an interval $\left\{z=t e^{ \pm i q}: 0 \leq t \leq 1\right\}$. Therefore, (32) defines a function which is analytic except the cut and, then, is an analytical continuation of $\Phi^{ \pm}$ from the outside of the unit disk to the whole complex plane.

Similarly, the following analytical continuations are obtained:

$$
\begin{aligned}
\Psi^{ \pm}(q, z) & =\frac{z e^{\mp i q}}{\Gamma(\beta)} \sum_{l=0}^{\infty} \rho_{l}^{ \pm}(q) \int_{0}^{\infty} d u \frac{u^{\beta-1} e^{-(l+1) u}}{z e^{\mp i q}-e^{-u}} \\
Z(z) & =\frac{z}{\Gamma(\beta)} \int_{0}^{\infty} d s \frac{s^{\beta-1} e^{-s}}{z-e^{-s}},
\end{aligned}
$$

both of which are analytic in the unit disk $|z|<1$ except the cuts. The function $\Psi^{ \pm}(q, z)$ has a cut on an interval $\left\{z=t e^{ \pm i q}: 0 \leq t \leq 1\right\}$ and $Z(z)$ has it on a real interval $[0,1]$. In addition, the first two terms of $(25)$ have the following analytical continuation:

$$
\begin{aligned}
\Xi^{ \pm}(q, z)+\frac{B_{1}^{ \pm}(q, 0) \Psi^{ \pm}(q, z)}{z}= & \frac{\Psi^{ \pm}(q, z)}{z} \sum_{l=1}^{\infty} l^{\beta} B_{l}^{ \pm}(q, 0)\left(z e^{\mp i q}\right)^{l-1} \\
& -\sum_{l=2}^{\infty} \sum_{k=1}^{l-1} \frac{l^{\beta} B_{l}^{ \pm}(q, 0) \widetilde{\rho}_{k}^{ \pm}(q)}{k^{\beta} e^{ \pm i q(l-k)}} z^{l-k-1}
\end{aligned}
$$

The series of the right-hand side are absolutely convergent for $|z|<1$ and, thus, analytic there. Note that (36) is not a simple rewrite since the series in (26) does not converge for $|z|<1$.

As a result, the integrand of (31) is meromorphic in the unit circle except the cuts. Its poles, if they exist, are given as zeros of $\Omega(q, z)$. When $q=0$, $\Omega(0, z)=0$ is equivalent to $Z(z) / z \pm \zeta(\beta)=0$. As easily seen, $Z(z) / z-\zeta(\beta)=$ 0 has a unique zero at $z=1$ and an argument similar to that given in Ref.[11] shows that $Z(z) / z+\zeta(\beta)=0$ has a unique real zero on $(-1,0)$. Or $\Omega(0, z)$ has two real zeros; one is in the interval $[-1,0]$ and the other at 1 . When $q \neq 0$, the behavior of $\Omega(q, z)$ is investigated numerically with the aid of Newton's method and we found that $\Omega(q, z)$ may have, at most, two simple real zeros; one in the interval $[-1,0]$ and the other in $[0,1]$, which we denote, respectively, as $\lambda_{d}^{-}(q)$ and $\lambda_{d}^{+}(q)$. Their properties will be discussed in Sec.4 again. 


\subsection{Decomposition of averages of observables}

Now we go back to the contour integral in (31). Note that, because of the $q$-integration, it is sufficient to consider $q \neq 0$ terms. Then, the arguments of the previous subsection show that, for each $q$, the integrand is analytic in $|z|<1$ except the simple poles at $z=\lambda_{d}^{ \pm}(q)$ and cuts on the segments $\left\{z=t e^{ \pm i q}: 0 \leq t \leq 1\right\}$. Therefore, the contour integral is evaluated by deforming the contour from the circle $|z|=r$ to the counter-clockwise curve $C(q)$ shown in Fig.2 and one gets

$$
\oint_{|z|=r} \frac{d z}{2 \pi i} z^{t} R(q, z)=\sum_{\sigma= \pm} \operatorname{Res}_{\lambda_{d}^{\sigma}} z^{t} R(q, z)+\oint_{C(q)} \frac{d z}{2 \pi i} z^{t} R(q, z)
$$

where $\lambda_{d}^{ \pm}(q)$ are abbreviated as $\lambda_{d}^{ \pm}$and $\operatorname{Res}_{\lambda_{d}^{ \pm}}$stands for the residue at $z=\lambda_{d}^{ \pm}$. Firstly, we consider the contribution from the curve $C(q)$ in case of $q>0$, which consists of two counter-clockwise $\operatorname{arcs} \gamma_{0 R}=\{z:|z|=\epsilon \&|\arg z|<|q|\}$ and $\gamma_{0 L}=\{z:|z|=\epsilon \&|\pi-\arg z|<\pi-|q|\}$, two counter-clockwise circles $\gamma_{ \pm}=\left\{z:\left|z-e^{ \pm i q}\right|=\epsilon\right\}$, the segment $C_{+l}^{\epsilon}=\left\{z=(t-i 0) e^{i q}: \epsilon \leq t \leq 1-\epsilon\right\}$ $\left(C_{+u}^{\epsilon}=\left\{z=(t+i 0) e^{i q}: \epsilon \leq t \leq 1-\epsilon\right\}\right)$ just below (above) the upper cut and the segment $C_{-l}^{\epsilon}=\left\{z=(t-i 0) e^{-i q}: \epsilon \leq t \leq 1-\epsilon\right\}$ $\left(C_{-u}^{\epsilon}=\left\{z=(t+i 0) e^{-i q}: \epsilon \leq t \leq 1-\epsilon\right\}\right)$ just below (above) the lower cut:

$$
C(q)=\gamma_{0 R} \cup C_{+l}^{\epsilon} \cup \gamma_{+} \cup C_{+u}^{\epsilon} \cup \gamma_{0 L} \cup C_{-l}^{\epsilon} \cup \gamma_{-} \cup C_{-u}^{\epsilon} .
$$

Here, $\epsilon$ stands for a small positive number, $C_{+u}^{\epsilon}$ and $C_{-u}^{\epsilon}$ point to the origin, and $C_{+l}^{\epsilon}$ and $C_{-l}^{\epsilon}$ point to the infinity.
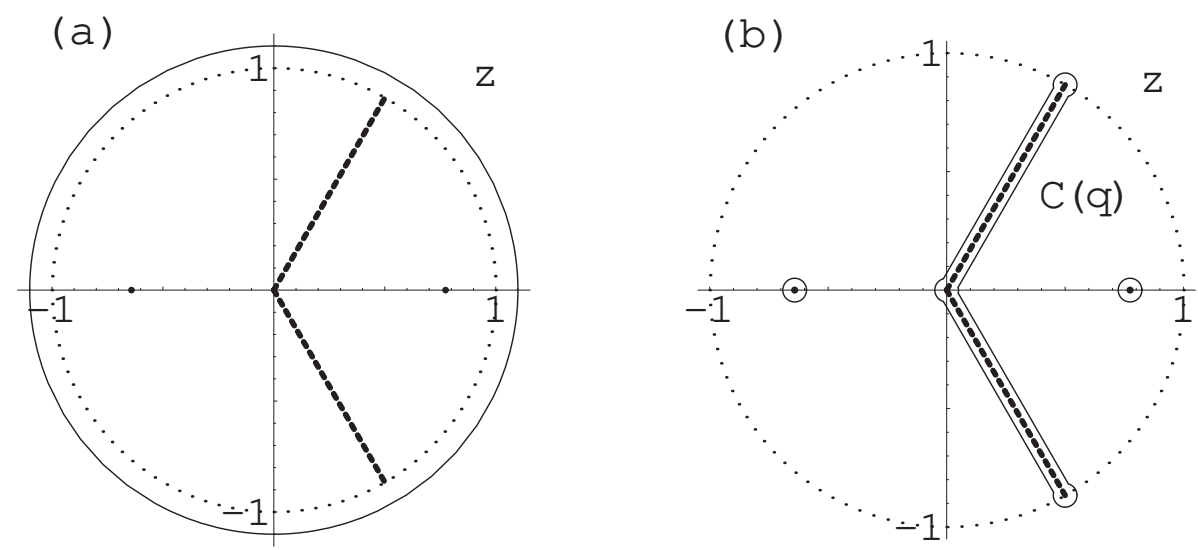

Fig. 2. Integration contours (solid curves) for individual $q$. Two dots and dotted lines indicate, respectively, the simple poles $\lambda_{d}^{ \pm}(q)$ and the cuts of the integrand. (a) The original circle $|z|=r(>1)$ and (b) the deformed contour consisting of two circles and the curve $C(q)$ surrounding two poles and the cuts, respectively. The directions are counter-clockwise and the unit circle is shown as a reference. 
Since the conditions $A \in X_{O}$ and $\rho \in X_{D}$ lead to

$$
\lim _{z \rightarrow 0}|z|^{\alpha}|R(q, z)|=0 \quad(0<\exists \alpha<1)
$$

the contributions from $\gamma_{0 R}$ and $\gamma_{0 L}$ vanish in the limit of $\epsilon \rightarrow 0$. Similarly, the contributions from $\gamma_{ \pm}$vanish in the same limit since

$$
\lim _{z \rightarrow e^{ \pm i q}}\left|z-e^{ \pm i q}\right|^{\alpha^{\prime}}|R(q, z)|=0 \quad\left(\min (2-\beta, 0)<\alpha^{\prime}<1\right)
$$

On the other hand, we have

$$
\lim _{\epsilon \rightarrow 0} \int_{C_{+l}^{\epsilon} \cup C_{+u}^{\epsilon}} \frac{d z}{2 \pi i} z^{t} R(q, z)=\int_{C_{q}^{+}} \frac{d z}{2 \pi i} z^{t}\left\{R\left(q, z_{l}^{+}\right)-R\left(q, z_{u}^{+}\right)\right\},
$$

where $C_{q}^{+} \equiv\left\{z=t e^{i q}: 0 \leq t \leq 1\right\}$ is the upper cut and $z_{u(l)}^{+}=z+(-) i e^{i q} 0$. Eqs.(25), (32) (34) and (35) lead to

$$
\begin{aligned}
& \frac{R\left(q, z_{l}^{+}\right)-R\left(q, z_{u}^{+}\right)}{2 \pi i}=N_{+}(q, z) {\left[\Delta \Psi^{+}(q, z)+\frac{\Psi^{-}(q, z)}{z e^{i q} \zeta(\beta)}\right] } \\
& \times z e^{-i q}\left[\Delta \Phi^{+}(q, z)+\frac{\Phi^{-}(q, z)}{z e^{-i q} \zeta(\beta)}\right]
\end{aligned}
$$

where

$$
\begin{aligned}
N_{+}(q, z) & =\frac{\left[-\ln \left(z e^{-i q}\right)\right]^{\beta-1}}{\Gamma(\beta) \Omega\left(q, z_{l}^{+}\right) \Omega\left(q, z_{u}^{+}\right)} \\
\Delta \Psi^{+}(q, z) & =\frac{\Gamma(\beta)\left[\Omega\left(q, z_{u}^{+}\right) \Psi^{+}\left(q, z_{l}^{+}\right)-\Omega\left(q, z_{l}^{+}\right) \Psi^{+}\left(q, z_{u}^{+}\right)\right]}{2 \pi i z e^{-i q}\left[-\ln \left(z e^{-i q}\right)\right]^{\beta-1}} \\
\Delta \Phi^{+}(q, z) & =\frac{\Gamma(\beta)\left[\Omega\left(q, z_{u}^{+}\right) \Phi^{+}\left(q, z_{l}^{+}\right)-\Omega\left(q, z_{l}^{+}\right) \Phi^{+}\left(q, z_{u}^{+}\right)\right]}{2 \pi i z e^{-i q}\left[-\ln \left(z e^{-i q}\right)\right]^{\beta-1}} .
\end{aligned}
$$

The contributions from the cut $C_{q}^{+}$with $q<0$ as well as from the other cut $C_{q}^{-} \equiv\left\{z=t e^{-i q}: 0 \leq t \leq 1\right\}$ are evaluated in a similar way and finally one obtains

$$
\lim _{\epsilon \rightarrow 0} \int_{C_{ \pm l}^{\epsilon} \cup C_{ \pm u}^{\epsilon}} \frac{d z}{2 \pi i} z^{t} R(q, z)=\int_{C_{q}^{ \pm}} d z\left(A, F_{ \pm}(q, z)\right) z^{t}\left(\widetilde{F}_{ \pm}(q, z), \rho\right)
$$

The functional $\left(A, F_{ \pm}(q, z)\right)$ is defined by 


$$
\begin{gathered}
\left(A, F_{ \pm}(q, z)\right)=\int_{0}^{1} d y \hat{A}(q, y)^{*} g_{q z}^{ \pm}(y) \\
g_{q z}^{ \pm}(y)=\sum_{l=1}^{\infty} \frac{e^{ \pm i q} l^{\beta} \chi_{l}^{\mp}(y)}{\zeta(\beta) \Gamma(\beta)} \int_{0}^{\infty} d s \frac{s^{\beta-1} e^{-l s}}{z e^{ \pm i q}-e^{-s}}+\frac{\Omega_{q z}^{ \pm}}{z} \sum_{l=1}^{\infty} l^{\beta} \chi_{l}^{ \pm}(y)\left(z e^{\mp i q}\right)^{l} \\
\quad+\sum_{l=1}^{\infty} l^{\beta} \chi_{l}^{ \pm}(y) \frac{e^{\mp i q} Z\left(z e^{ \pm i q}\right)}{z e^{ \pm i q} \zeta(\beta)^{2} \Gamma(\beta)} \int_{0}^{\infty} d s \mathcal{P} \frac{s^{\beta-1} e^{-l s}}{z e^{\mp i q}-e^{-s}}, \\
\chi_{l}^{-}(y)=\chi_{l}^{+}(1-y)= \begin{cases}1 & \left(\xi_{l}<y \leq \xi_{l-1}\right) \\
0 & (\text { otherwise })\end{cases} \\
\Omega_{q z}^{ \pm}=1-\frac{Z\left(z e^{ \pm i q}\right)}{z e^{ \pm i q} \zeta(\beta)^{2} \Gamma(\beta)} \int_{0}^{\infty} d s \mathcal{P} \frac{s^{\beta-1} e^{-s}}{z e^{\mp i q}-e^{-s}}
\end{gathered}
$$

where $\mathcal{P}$ stands for Cauchy's principal value. Note that $z e^{\mp i q}$ is real if $z \in C_{q}^{ \pm}$. Similarly, $\left(\widetilde{F}_{ \pm}(q, z), \rho\right)$ is given by

$$
\begin{gathered}
\left(\widetilde{F}_{ \pm}(q, z), \rho\right)=n_{q z}^{ \pm}\left\{\sum_{j=0}^{\infty} \frac{\rho_{j}^{ \pm}(q) Z\left(z e^{ \pm i q}\right)}{z e^{ \pm i q} \zeta(\beta)^{2} \Gamma(\beta)} \int_{0}^{\infty} d s \mathcal{P} \frac{s^{\beta-1} e^{-(j+1) s}}{z e^{\mp i q}-e^{-s}}\right. \\
\left.+\Omega_{q z}^{ \pm} \sum_{j=0}^{\infty} \rho_{j}^{ \pm}(q)\left(z e^{\mp i q}\right)^{j}+\sum_{j=0}^{\infty} \frac{\rho_{j}^{\mp}(q)}{\zeta(\beta) \Gamma(\beta)} \int_{0}^{\infty} d s \frac{s^{\beta-1} e^{-(j+1) s}}{z e^{ \pm i q}-e^{-s}}\right\} \\
n_{q z}^{ \pm}=\frac{\left[-\log z e^{\mp i q}\right]^{\beta-1}}{\Gamma(\beta) \Omega\left(q, z+i e^{ \pm i q} 0\right) \Omega\left(q, z-i e^{ \pm i q} 0\right)}
\end{gathered}
$$

The pole contributions are calculated as above and one has

$$
\begin{aligned}
\sum_{\sigma= \pm} \operatorname{Res}_{\lambda_{d}^{\sigma}} z^{t} R(q, z) & =\sum_{\sigma= \pm} \lambda_{d}^{\sigma}(q)^{t} \lim _{z \rightarrow \lambda_{d}^{\sigma}(q)}\left(z-\lambda_{d}^{\sigma}(q)\right) R(q, z) \\
& =\sum_{\sigma= \pm}\left(A, F_{d}^{\sigma}(q)\right) \lambda_{d}^{\sigma}(q)^{t}\left(\widetilde{F}_{d}^{\sigma}(q), \rho\right)
\end{aligned}
$$

where the functionals are given by

$$
\begin{aligned}
\left(A, F_{d}^{\sigma}(q)\right)= & \int_{0}^{1} d y \hat{A}(q, y)^{*} g_{d}^{ \pm}(q, y) \\
g_{d}^{ \pm}(q, y)= & \sum_{l=1}^{\infty} \frac{Z\left(\lambda_{d}^{ \pm} e^{i q}\right) l^{\beta} \chi_{l}^{+}(y)}{\lambda_{d}^{ \pm} e^{i q} \zeta(\beta) \Gamma(\beta)} \int_{0}^{\infty} d s \frac{s^{\beta-1} e^{-l s}}{\lambda_{d}^{ \pm} e^{-i q}-e^{-s}} \\
& +\sum_{l=1}^{\infty} \frac{e^{2 i q} l^{\beta} \chi_{l}^{-}(y)}{\Gamma(\beta)} \int_{0}^{\infty} d s \frac{s^{\beta-1} e^{-l s}}{\lambda_{d}^{ \pm} e^{i q}-e^{-s}}
\end{aligned}
$$




$$
\begin{aligned}
\left(\widetilde{F}_{d}^{\sigma}(q), \rho\right)= & \sum_{j=0}^{\infty} \frac{e^{-i q} \rho_{j}^{+}(q)}{\Omega^{\prime}\left(q, \lambda_{d}^{ \pm}\right) \zeta(\beta) \Gamma(\beta)} \int_{0}^{\infty} d s \frac{s^{\beta-1} e^{-(j+1) s}}{\lambda_{d}^{ \pm} e^{-i q}-e^{-s}} \\
& +\sum_{j=0}^{\infty} \frac{\lambda_{d}^{ \pm} \rho_{j}^{-}(q)}{\Omega^{\prime}\left(q, \lambda_{d}^{ \pm}\right) Z\left(\lambda_{d}^{ \pm} e^{i q}\right) \Gamma(\beta)} \int_{0}^{\infty} d s \frac{s^{\beta-1} e^{-(j+1) s}}{\lambda_{d}^{ \pm} e^{i q}-e^{-s}}
\end{aligned}
$$

In the above, $\lambda_{d}^{ \pm}(q)$ is abbreviated as $\lambda_{d}^{ \pm}$and $\Omega^{\prime}(q, z)$ stands for the derivative of $\Omega(q, z)$ with respect to $z$.

In summary, we have derived

$$
\begin{aligned}
\left(A, \hat{P}^{t} \rho\right)= & \int_{-\pi}^{\pi} \frac{d q}{2 \pi} \sum_{\sigma= \pm}\left(A, F_{d}^{\sigma}(q)\right) \lambda_{d}^{\sigma}(q)^{t}\left(\widetilde{F}_{d}^{\sigma}(q), \rho\right) \\
& +\int_{-\pi}^{\pi} \frac{d q}{2 \pi} \sum_{\alpha= \pm} \int_{C_{q}^{\alpha}} d z\left(A, F_{\alpha}(q, z)\right) z^{t}\left(\widetilde{F}_{\alpha}(q, z), \rho\right)
\end{aligned}
$$

\subsection{Generalized eigenfunctions of the Frobenius-Perron operator}

We remark that, for any bounded linear functional $F$ over $X_{O},\left(\hat{P}^{*} A, F\right)(A \in$ $\left.X_{O}\right)$ is well defined due to the invariance of $X_{O}$ under $\hat{P}^{*}$ and that the operator $\hat{P}$ can be extended via $(A, \hat{P} F) \equiv\left(\hat{P}^{*} A, F\right)$ to the space of all bounded linear functionals over $X_{O}$ (the dual space of $X_{O}$ ). The linear functionals $F_{d}^{ \pm}$and $F_{ \pm}(q, z)$ are bounded and satisfy the following relations for any $A \in X_{O}$ :

$$
\begin{aligned}
\left(A, \hat{P} F_{d}^{ \pm}(q)\right) & \equiv\left(\hat{P}^{*} A, F_{d}^{ \pm}(q)\right)=\lambda_{d}^{ \pm}(q)\left(A, F_{d}^{ \pm}(q)\right) \\
\left(A, \hat{P} F_{ \pm}(q, z)\right) & \equiv\left(\hat{P}^{*} A, F_{ \pm}(q, z)\right)=z\left(A, F_{ \pm}(q, z)\right) . \quad\left(z \in C_{q}^{ \pm}\right)
\end{aligned}
$$

Namely, they are eigenfunctions of $\hat{P}$ in the generalized sense[19].

Similarly, the operator $\hat{P}^{*}$ can be extended to the dual space of $X_{D}$ and the functionals $\tilde{F}_{d}^{ \pm}, \tilde{F}_{l}(q, z), \tilde{F}_{u}(q, z)$ are its generalized eigenfunctions:

$$
\begin{aligned}
\left(\hat{P}^{*} \tilde{F}_{d}^{ \pm}(q), \rho\right) & =\left(\tilde{F}_{d}^{ \pm}(q), \hat{P} \rho\right)=\lambda_{d}^{ \pm}(q)\left(\tilde{F}_{d}^{ \pm}(q), \rho\right), \\
\left(\hat{P}^{*} \tilde{F}_{ \pm}(q, z), \rho\right) & =\left(\tilde{F}_{ \pm}(q, z), \hat{P} \rho\right)=z\left(\tilde{F}_{ \pm}(q, z), \rho\right), \quad\left(z \in C_{q}^{ \pm}\right)
\end{aligned}
$$

Sometimes, eigenfunctions of $\hat{P}$ and $\hat{P}^{*}$ are referred to, respectively, as right and left eigenfunctions of $\hat{P}$.

It is then interesting to reinterpret (54) from this point of view. One has 


$$
\begin{aligned}
(A, \rho)= & \int_{-\pi}^{\pi} \frac{d q}{2 \pi} \sum_{\sigma= \pm}\left(A, F_{d}^{\sigma}(q)\right)\left(\widetilde{F}_{d}^{\sigma}(q), \rho\right) \\
& +\int_{-\pi}^{\pi} \frac{d q}{2 \pi} \sum_{\alpha= \pm} \int_{C_{q}^{\alpha}} d z\left(A, F_{\alpha}(q, z)\right)\left(\widetilde{F}_{\alpha}(q, z), \rho\right) \\
(A, \hat{P} \rho)= & \int_{-\pi}^{\pi} \frac{d q}{2 \pi} \sum_{\sigma= \pm}\left(A, F_{d}^{\sigma}(q)\right) \lambda_{d}^{\sigma}(q)\left(\widetilde{F}_{d}^{\sigma}(q), \rho\right) \\
& +\int_{-\pi}^{\pi} \frac{d q}{2 \pi} \sum_{\alpha= \pm} \int_{C_{q}^{\alpha}} d z\left(A, F_{\alpha}(q, z)\right) z\left(\widetilde{F}_{\alpha}(q, z), \rho\right)
\end{aligned}
$$

The first relation (59) corresponds to the completeness of the set of left eigenfunctions $\left\{F_{d}^{ \pm}(q), F_{ \pm}(q, z)\right\}$ and that of right ones $\left\{\tilde{F}_{d}^{ \pm}(q), \tilde{F}_{ \pm}(q, z)\right\}$. The second relation (60) can be considered as a spectral decomposition of $\hat{P}$ in terms of those eigenfunctions, which correspond, for each $q$, to two isolated eigenvalues $\lambda_{d}^{ \pm}(q)$ and continuous spectra on $C_{q}^{ \pm}$. These relations are regarded as an extension to the FP operator of the generalized spectral decomposition in the sense of Ref.[19], which was originally formulated for self-adjoint and unitary operators. Such extended spectral decompositions are widely used to study statistical properties of dynamical systems (see $[2,7]$ and references therein).

\section{Transports}

For systems exhibiting deterministic diffusion with diffusion coefficient $D[2,3,6,7]$, the $\mathrm{FP}$ operator has an eigenvalue $z=z_{q}$ such that $z_{q} \simeq 1-D q^{2}$ for small wavenumber $|q|$ and it fully characterizes the diffusion in a scaling limit. A similar behavior is expected for the map $\widetilde{\phi}$. We then investigate an eigenvalue $\lambda_{d}^{+}(q)$ for $|q| \ll 1$ and appropriate scaling limits.

\subsection{Case of $\beta>3$}

When $|q| \ll 1,\left|1-\lambda_{d}^{+} e^{ \pm i q}\right| \ll 1$ and it is sufficient to retain the largest terms of $\Omega(q, z)$ with respect to $\left|1-z e^{ \pm i q}\right|$. For $\beta>3$, they are found to be

$$
\begin{aligned}
\Omega(q, z) \simeq & \frac{2 \zeta(\beta-1)}{\zeta(\beta)}(z \cos q-1)-\left(\frac{\zeta(\beta-1)}{\zeta(\beta)}\right)^{2}\left(1+z^{2}-2 z \cos q\right) \\
& -\frac{\zeta(\beta-1)+\zeta(\beta-2)}{\zeta(\beta)}\left(1-2 z \cos q+z^{2} \cos 2 q\right) .
\end{aligned}
$$



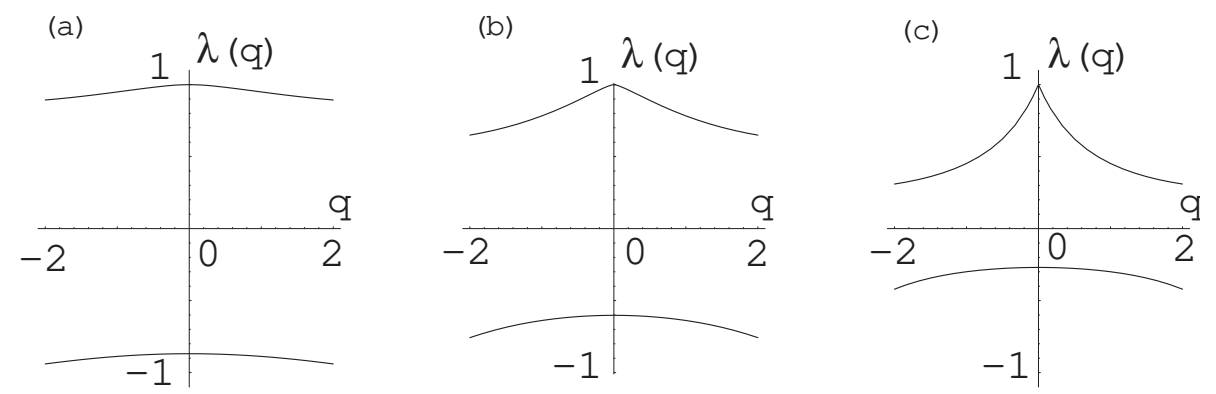

Fig. 3. The $q$-dependence of the eigenvalues of the FP operator. The upper branch is $\lambda_{d}^{+}(q)$ and the lower branch is $\lambda_{d}^{-}(q)$. (a) $\beta=4$, (b) $\beta=2.5$ and (c) $\beta=1.7$.

Then, the equation $\Omega\left(q, \lambda_{d}^{+}\right)=0$ has a solution

$$
\lambda_{d}^{+} \simeq 1-\frac{1}{2}\left[\frac{\zeta(\beta-2)}{\zeta(\beta-1)}-\frac{\zeta(\beta-1)}{\zeta(\beta)}\right] q^{2},
$$

which implies that the map exhibits ordinary diffusion. In Fig.3 (a), the $q$ dependence of numerically calculated $\lambda_{d}^{ \pm}(q)$ for $\beta=4$ is shown. The $q^{2}-$ behavior of $\lambda_{d}^{+}(q)$ near $q=0$ is clearly seen.

The appearance of ordinary diffusion can be seen more explicitly by considering a scaling limit, where one sets $n=L_{\xi} X, t=L_{\tau} T$ and the limit of $L_{\xi} \rightarrow+\infty$ is taken while assuming a functional relation between $L_{\xi}$ and $L_{\tau}$. Accordingly, the observable $A\left(L_{\xi} X\right)$ is assumed to be a well-behaved function of $X$. Then, one has

$$
\frac{1}{L_{\xi}} \hat{A}\left(Q / L_{\xi}\right) \equiv \frac{1}{L_{\xi}} \sum_{n=-\infty}^{\infty} e^{i n Q / L_{\xi}} A(n) \simeq \hat{A}^{(\mathrm{cg})}(Q)
$$

where $\hat{A}^{(\mathrm{cg})}(Q) \equiv \int_{-\infty}^{\infty} d X e^{i Q X} A\left(L_{\xi} X\right)$ is the Fourier transform of the coarsegrained observable. Similarly, the Fourier transform of the probability distribution is assumed to be constant on each unit interval and is a slowly varying function of the site coordinate $[x]$. Then, in terms of the Fourier transform of the coarse-grained density $\hat{\rho}^{(\mathrm{cg})}$, one has $\rho_{l}^{ \pm}\left(Q / L_{\xi}\right)=\delta_{l, 0} \hat{\rho}^{(\mathrm{cg})}(Q)$. Hence,

$$
\begin{aligned}
& \left(A, \hat{P}^{L_{\tau} T} \rho\right) \simeq \int_{-\infty}^{\infty} \frac{d Q}{2 \pi} \hat{A}^{(\mathrm{cg})}(Q)^{*} \hat{\rho}^{(\mathrm{cg})}(Q)\left\{\sum_{\sigma= \pm}\left(e, F_{d}^{\sigma}(q)\right) \lambda_{d}^{\sigma}(q)^{L_{\tau} T}\left(\widetilde{F}_{d}^{\sigma}(q), e\right)\right. \\
& \left.+\sum_{\alpha= \pm} e^{\alpha i q\left(L_{\tau} T+1\right)} \int_{0}^{1} d s\left(e, F_{\alpha}\left(q, s e^{\alpha i q}\right)\right) s^{L_{\tau} T}\left(\widetilde{F}_{\alpha}\left(q, s e^{\alpha i q}\right), e\right)\right\}_{q=\frac{Q}{L_{\xi}}}
\end{aligned}
$$

where $e$ is a function of $n$ and $y$ with the Fourier transform $\hat{e}(q, y)=1$. In order to have a non-trivial scaling limit, one must have $L_{\tau} \propto L_{\xi}^{2}$ and here we 
take $L_{\tau}=L_{\xi}^{2}$. Then, one has

$$
\lim _{\substack{L_{\xi} \rightarrow \infty \\ L_{\tau}=L_{\xi}^{2}}}\left(e, F_{d}^{+}\left(\frac{Q}{L_{\xi}}\right)\right) \lambda_{d}^{+}\left(\frac{Q}{L_{\xi}}\right)^{L_{\tau} T}\left(\widetilde{F}_{d}^{+}\left(\frac{Q}{L_{\xi}}\right), e\right)=e^{-D_{\beta}^{N} Q^{2} T}
$$

where $D_{\beta}^{N}=\frac{1}{2}\left[\frac{\zeta(\beta-2)}{\zeta(\beta-1)}-\frac{\zeta(\beta-1)}{\zeta(\beta)}\right]$ is the diffusion coefficient. In this scaling limit, the contribution from $\lambda_{d}^{-}$vanishes as $\sup _{q}\left|\lambda_{d}^{-}(q)\right|<1$. This is also the case for the continuous spectrum as $\left.k(s, Q) \equiv \lim _{L_{\xi} \rightarrow \infty}\left(e, F_{\alpha}\left(q, s e^{\alpha i q}\right)\right)\left(\widetilde{F}_{\alpha}\left(q, s e^{\alpha i q}\right), e\right)\right|_{q=\frac{Q}{L_{\xi}}}$ is a bounded function of $s$ :

$$
\begin{aligned}
& \lim _{\substack{L_{\xi} \rightarrow \infty \\
L_{\tau}=L_{\xi}^{2}}}\left|\int_{0}^{1} d s\left(e, F_{\alpha}\left(\frac{Q}{L_{\xi}}, s e^{\alpha i q}\right)\right) s^{L_{\tau} T}\left(\widetilde{F}_{\alpha}\left(\frac{Q}{L_{\xi}}, s e^{\alpha i q}\right), e\right)\right| \\
& \quad \leq \sup _{s, Q}|k(s, Q)| \lim _{L_{\tau} \rightarrow \infty} \int_{0}^{1} d s s^{L_{\tau} T}=0 .
\end{aligned}
$$

In short, the scaling limit of the average value $\langle A\rangle_{T} \equiv \lim _{L_{\xi} \rightarrow \infty}\left\langle A, \hat{P}^{L_{\tau} T} \rho\right\rangle$ of $A$ at time $T$ is given by

$$
\langle A\rangle_{T}=\int_{-\infty}^{\infty} \frac{d Q}{2 \pi} \hat{A}^{(\mathrm{cg})}(Q)^{*} e^{-D_{\beta}^{N} Q^{2} T} \hat{\rho}^{(\mathrm{cg})}(Q)
$$

which is nothing but the solution of the diffusion equation.

\subsection{Case of $3 \geq \beta>2$}

When $\beta=3$, the largest terms of $\Omega(q, z)$ with respect to $\left|1-z e^{ \pm i q}\right|$ are

$$
\Omega(q, z) \simeq \frac{2 \zeta(2)}{\zeta(3)}(z \cos q-1)+\sum_{\sigma= \pm} \frac{\left(1-z e^{\sigma i q}\right)^{2} \log \left(1-z e^{\sigma i q}\right)}{2 \zeta(3)}
$$

from which one obtains the following solution of $\Omega\left(q, \lambda_{d}^{+}\right)=0$ :

$$
\lambda_{d}^{+} \simeq 1-\frac{q^{2}\left|\log q^{2}\right|}{4 \zeta(2)} .
$$

Similarly, when $2<\beta<3$, the leading term of $\Omega(q, z)$ is 


$$
\Omega(q, z) \simeq \frac{2 \zeta(\beta-1)}{\zeta(\beta)}(z \cos q-1)+\sum_{\sigma= \pm} \frac{\pi e^{-i \pi \beta}\left(1-z e^{\sigma i q}\right)^{\beta-1}}{\Gamma(\beta) \zeta(\beta) \sin \pi \beta}
$$

and the approximate solution of $\Omega\left(q, \lambda_{d}^{+}\right)=0$ is

$$
\lambda_{d}^{+} \simeq 1-\frac{\pi|q|^{\beta-1}}{2 \zeta(\beta-1) \Gamma(\beta)\left|\cos \frac{\pi \beta}{2}\right|} .
$$

In both cases, the system exhibits anomalous diffusion. The $q$-dependence of numerically calculated $\lambda_{d}^{ \pm}$for $\beta=2.5$ is shown in Fig.3 (b) and a $q$-dependence different from that for the normal diffusion is seen in the figure.

Now we consider a scaling limit for $2<\beta<3$, where we set $n=L_{\xi} X$, $t=L_{\tau} T$ and take the limit of $L_{\xi} \rightarrow+\infty$ while keeping $L_{\tau}=L_{\xi}^{\beta-1}$. Then, the contributions from the eigenvalue $\lambda_{d}^{-}$and continuous spectrum vanish because of the same reason as in the previous subsection, and in terms of the coarse-grained observable $\hat{A}^{(\mathrm{cg})}(Q)$ and probability density $\hat{\rho}^{(\mathrm{cg})}(Q)$ introduced previously, one has

$$
\langle A\rangle_{T} \equiv \lim _{\substack{L_{\xi} \rightarrow \infty \\ L_{\tau}=L_{\xi}^{\beta-1}}}\left\langle A, \hat{P}^{L_{\tau} T} \rho\right\rangle=\int_{-\infty}^{\infty} \frac{d Q}{2 \pi} \hat{A}^{(\mathrm{cg})}(Q)^{*} e^{-D_{\beta}^{L}|Q|^{\beta-1} T} \hat{\rho}^{(\mathrm{cg})}(Q)
$$

where $D_{\beta}^{L}=\frac{\pi}{2 \zeta(\beta-1) \Gamma(\beta)\left|\cos \frac{\pi \beta}{2}\right|}$.

\subsection{Case of $2 \geq \beta>1$}

When $\beta=2$, the leading term of $\Omega(q, z)$ with respect to $\left|1-z e^{ \pm i q}\right|$ is

$$
\Omega(q, z) \simeq \frac{1-z e^{i q}}{\zeta(2)} \log \frac{1-z e^{i q}}{-z e^{i q+1}}+\frac{1-z e^{-i q}}{\zeta(2)} \log \frac{1-z e^{-i q}}{-z e^{-i q+1}}
$$

By setting $-z e^{-i q}=e^{i \pi}+r e^{i \theta}$ and $-z e^{i q}=e^{i \pi}+r e^{i(2 \pi-\theta)}(0<\theta<2 \pi)$, one finds the approximate solution of $\Omega\left(q, \lambda_{d}^{+}\right)=0$ :

$$
\lambda_{d}^{+} \simeq 1-\frac{\pi|q|}{2|\log | q||} \cdot \quad(|q| \ll 1)
$$

On the other hand, when $1<\beta<2$, the leading term of $\Omega(q, z)$ is 


$$
\Omega(q, z) \simeq \frac{-\pi e^{-i \pi \beta}}{\zeta(\beta) \Gamma(\beta)|\sin \pi \beta|}\left\{\left(1-z e^{i q}\right)^{\beta-1}+\left(1-z e^{-i q}\right)^{\beta-1}\right\}
$$

and, by the same way as above, one finds that $\Omega\left(q, \lambda_{d}^{+}\right)=0$ admits a solution

$$
\lambda_{d}^{+}=\frac{\sin \left(\frac{\pi}{2(\beta-1)}\right)}{\sin \left(\frac{\pi}{2(\beta-1)}-|q|\right)} \simeq 1-\left|\cot \frac{\pi}{2(\beta-1)}\right||q|, \quad(|q| \ll 1)
$$

when $3 / 2<\beta<2$ and has no solution when $\beta \leq 3 / 2$. The $q$-dependence of numerically calculated $\lambda_{d}^{ \pm}$for $\beta=1.7$ is shown in Fig.3 (c), where $|q|$-linear behavior of $\lambda_{d}^{+}$is seen near $q=0$. Moreover, for $\beta=1.1$, Newton's method fails to find a solution of $\Omega(q, z)=0$ and this is consistent with the absence of the solution.

Finally, we consider a scaling limit for $1<\beta<2$, where we set $n=L_{\xi} X$, $t=L_{\tau} T$ and take the limit of $L_{\xi} \rightarrow+\infty$ while keeping $L_{\tau}=L_{\xi}$. As before, the contribution from the eigenvalue $\lambda_{d}^{-}$vanishes. In this case, however, the continuous spectra do contribute and, after a tedious but straightforward calculation, one obtains

$$
\begin{aligned}
\langle A\rangle_{T} \equiv \lim _{\substack{L_{\xi} \rightarrow \infty \\
L_{\tau}=L_{\xi}}}\left\langle A, \hat{P}^{L_{\tau} T} \rho\right\rangle= & \int_{-\infty}^{\infty} \frac{d Q}{2 \pi} \hat{A}^{(\mathrm{cg})}(Q)^{*} \hat{\rho}^{(\mathrm{cg})}(Q)\{\cos Q T \\
& \left.+\int_{\Gamma} \frac{d z}{2 \pi} \frac{e^{-|Q| T z}}{z^{2}+1} \frac{(z-i)^{\beta-1}-(z+i)^{\beta-1}}{(z-i)^{\beta-1}+(z+i)^{\beta-1}}\right\}
\end{aligned}
$$

where $\Gamma=\{z=t+i(1+\epsilon) \mid t:+\infty \rightarrow 0\} \cup\left\{z=(1+\epsilon) e^{i \theta} \mid \theta: \frac{\pi}{2} \rightarrow \frac{3 \pi}{2}\right\} \cup$ $\{z=t-i(1+\epsilon) \mid t: 0 \rightarrow+\infty\}$ is the integration contour with $\epsilon$ an arbitrary positive constant, and the branch cut of $(z \mp i)^{\beta-1}$ is $\{z=t \pm i \mid t: 0 \rightarrow+\infty\}$.

\section{CONCLUSIONS}

We have studied, for a piecewise linear approximation of Geisel-NierwetbergZacherl map, the evolution of statistical averages of bounded observables which are differentiable at the origin with respect to piecewise constant initial densities which have certain smoothness at the origin. Because of the periodicity of the system, the average value is expressed by a superposition of Fourier components and the time evolution of each component is governed by two simple eigenvalues $\lambda_{d}^{ \pm}(q)$ and continuous spectra $C_{q}^{ \pm}=\left\{z=t e^{ \pm i q}: 0 \leq t \leq 1\right\}$ of the FP operator. The corresponding generalized eigenfunctions are explicitly constructed. 
The main difference from the hyperbolic systems with deterministic diffusion is the existence of the continuous spectra starting from the unit circle for each wavenumber $q$. In spite of this fact, when $\beta>2$, the transport in an appropriate scaling limit is entirely controlled by a simple eigenvalue $\lambda_{d}^{+}(q)$ located near 1 and the average value of the coarse-grained description $\langle A\rangle_{T}$ obeys

$$
\langle A\rangle_{T}=\int_{-\infty}^{\infty} \frac{d Q}{2 \pi} \hat{A}^{(\mathrm{cg})}(Q)^{*} e^{-D_{\beta}|Q|^{\mu} T} \hat{\rho}^{(\mathrm{cg})}(Q)
$$

where $\mu=2$ and $D_{\beta}=D_{\beta}^{N}$ for $\beta>3$, and $\mu=\beta-1$ and $D_{\beta}=D_{\beta}^{L}$ for $3>\beta>2$. In other words, when the reduced map is in the stationary regime, transports in the scaling limits are governed by the eigenvalues. And the normal diffusion appears if the fluctuations are normal and the Lévy flight appears if the fluctuations are of Lévy-type.

Here a remark is in order. As is well known, in periodic maps with marginal fixed points of Geisel-Nierwetberg-Zacherl type, the mean square displacement is well-defined and grows faster than the normal diffusion. Indeed, with the aid of (54), one recovers a known result on the mean square displacement:

$$
\left\langle\left([x]-\langle[x]\rangle_{t}\right)^{2}\right\rangle_{t} \propto\left\{\begin{array}{ll}
t & \beta>3 \\
t \log t & \beta=3 \\
t^{4-\beta} & 3>\beta>2 \\
t^{2} & 2>\beta>1
\end{array},\right.
$$

where $\langle\cdots\rangle_{t}$ is the average at time $t$ with respect to an initial distribution $\rho(x)=\delta_{[x], n_{0}}$ for some $n_{0}$. On the other hand, the mean square displacement is ill-defined for Lévy flights[20]. However, there is no contradiction between the two conclusions because the ill-definedness of the mean square displacement comes from the scaling limit.

The situation changed for $2>\beta>1$ or when the reduced map is in the non-stationary regime. Then the 'macroscopic' transport is described by (77), the first term of which represents ballistic transport, while the second term corresponds to a more complicated process.

Note that the space $X_{D}$ of initial densities is not dense in $L^{2}[0,1]$ and we will discuss elsewhere a complementary description in terms of initial densities which form a dense subset of $L^{2}[0,1]$. 


\section{Acknowledgments}

The authors are grateful to Professors Y. Aizawa, J. Klafter and R. Klages

for fruitful discussions and comments. P.G. is financially supported by the National Fund for Scientific Research (FNRS Belgium). This work is supported by a Grant-in-Aid for Scientific Research (C) from JSPS and by a Grant-inAid for Scientific Research of Priority Areas "Control of Molecules in Intense Laser Fields" from the Ministry of Education, Culture, Sports, Science and Technology of Japan.

\section{References}

[1] D. Ruelle, Thermodynamic Formalism, (Addison-Wesley, Reading, 1987).

[2] P. Gaspard, Chaos, scattering and statistical mechanics, (Cambridge Univ. Press, Cambridge, 1998).

[3] J.R. Dorfman, An Introduction to Chaos in Non-Equilibrium Statistical Mechanics, (Cambridge Univ. Press, Cambridge, 1999).

[4] M. Pollicott, Invent. Math. 81 (1985) 413; Ann. Math. 131 (1990).

[5] D. Ruelle, Phys. Rev. Lett. 56 (1986) 405; J. Stat. Phys. 44 (1986) 281; J. Diff. Geom. 25 (1987) 99; Commun. Math. Phys. 125 (1989) 239; Publ. Math. IHES 72 (1990) 175.

[6] T. Tél and J. Vollmer, in Hard Ball Systems and the Lorentz Gas, ed. D. Szász, (Encyclopedia of Mathematical Science, 101, Springer, Berlin, 2000).

[7] S. Tasaki, Adv. Chem. Phys. 122 (2002) 77: P. Gaspard, Adv. Chem. Phys. 122 (2002) 109.

[8] T. Gilbert, J.R. Dorfman and P. Gaspard, Phys. Rev. Lett. 85 (2000) 1606; Nonlinearity 14 (2001) 339.

[9] R. Artuso, G. Casati, and R. Lombardi, Phys. Rev. Lett. 71 (1993) 62; X.-J. Wang and C.-K. Hu, Phys. Rev. E 48 (1993) 728.

[10] R. Artuso, Phys. Rep. 290 (1997) 37.

[11] S. Tasaki and P. Gaspard, J. of Stat. Phys., 109 (2002) 803.

[12] P. Manneville and Y. Pomeau, Phys. Lett. 75A (1979) 1; Y. Pomeau and P. Manneville, Commun. Math. Phys. 14 (1980) 189.

[13] T. Geisel, J. Nierwetberg and A. Zacherl, Phys. Rev. Lett. 54 (1985) 616. 
[14] T. Geisel and S. Thomae, Phys. Rev. Lett. 52 (1984) 1936.

[15] J.P. Bouchaud and A. Georges, Phys. Rep. 195 (1990) 127; R. Metzler and J. Klafter, Adv. Chem. Phys. 116 (2001) 223; Lévy Flights and Related Topics in Physics, edited by M.F. Shlesinger, G.M. Zaslavsky and U. Frisch, (Springer, Berlin, 1994).

[16] J. Dräger and J. Klafter, Phys. Rev. Lett. 84 (2000) 5998.

[17] T. Miyaguchi and Y. Aizawa, Prog. Theor. Phys. 106 (2001) 697.

[18] P. Gaspard and X.-J. Wang, Proc. Nat. Acad. Sci. USA 85 (1988) 4591; X.-J. Wang, Phys. Rev. A 39 (1989) 3214; X.-J. Wang, Phys. Rev. A 40 (1989) 6647.

[19] I. Gelfand and G. Shilov, Generalized Functions, Vol. 3 (Academic Press, New York, 1967); I. Gelfand and N. Vilenkin, Generalized Functions, Vol. 4 (Academic Press, New York, 1964); K. Maurin, General Eigenfunction Expansion and Unitary Representations of Topological Groups, (Polish Sci. Publ., Warszawa, 1968); G. Lindblad and B. Nagel, Ann. Inst. H. Poincaré 13, (1970) 27.

[20] P. Lévy, Theorie de l'addition des variables aléatoires (Gauthier-Villars, Paris, 1937); V. Seshadri and B.J. West, Proc. Natl. Acad. Sci. USA, 79 (1982) 4501. 